\title{
Current Evidence on Complete Digital Workflow in Implant Prosthodontics
}

\author{
Sunil K Mishra ${ }^{1}$, Ramesh Chowdhary ${ }^{2}$ \\ International Journal of Prosthodontics and Restorative Dentistry (2021): 10.5005/jp-journals-10019-1324
}

The successful outcome of implant treatment depends on accurate prosthetically driven implant placement. The three-dimensional (3D) imaging systems; cone-beam computed tomography (CBCT), 3D implant planning software, and computer-aided design/ computer-assisted manufacturing (CAD/CAM) systems have enhanced the treatment outcome of the dental implants. ${ }^{1}$

The digital workflow in fixed implant prosthodontics can be a partial-digital workflow or a complete digital workflow. The complete digital workflow is considered as a sequence of procedures using only virtual imaging and designing for the surgical planning of implant and guide fabrication with CAD/ CAM. ${ }^{2,3}$ The final implant prosthesis is fabricated in a monolithic design from lithium disilicate, zirconia, or hybrid ceramic materials. Currently, many researchers have evaluated the accuracy of implant placement following the complete digital workflow. ${ }^{2,4-6}$

Lin et al. ${ }^{4}$ had evaluated the clinical accuracy of static-guided implant surgery (sGIS) utilizing a complete digital workflow in 21 partially edentulous patients. They superimposed pre- and postoperative CBCT images and studied the positional and angular deviations between planned and placed implants using metrology software. They found that the clinical accuracy of the sGIS planned with a complete digital workflow was accordant with the conventional workflow. In another trial, Gintaute et al. ${ }^{5}$ had done a randomized controlled trial in 20 partially edentulous subjects to analyze the technical and clinical performance of monolithic zirconium oxide implant-fixed dental prostheses (iFDPs) fabricated using complete digital workflows and one mixed analog-digital workflow. They found that all tested workflows were feasible for treatment with iFDPs but chairside adjustments may be necessary, at least in every second patient, independent of the workflow used.

Michelinakis et al. ${ }^{6}$ in their review article had presented an overview of the current evidence on the direct digital workflow in patients rehabilitated with implant-supported prostheses. The vast majority of identified studies were in vitro and this limited their clinical significance. Based on the available literature, they found that single-implant monolithic restorations fabricated with a complete digital workflow had shown promising results in 3-5 years of follow-up. Siqueira et al. ${ }^{2}$ had done a systematic review of clinical trials in partially edentulous subjects comparing the accuracy of fully-guided static computer-assisted implant surgery (s-CAIS) following partial and complete digital workflows. A total of 669 implants were placed in 325 patients using s-CAIS and found that the accuracy was similar in both partial and complete digital workflows.
${ }^{1}$ Department of Prosthodontics, Rama Dental College, Hospital and Research Centre, Kanpur, Uttar Pradesh, India

${ }^{2}$ Department of Prosthodontics, RajaRajeswari Dental College and Hospital, Bengaluru, Karnataka, India

Corresponding Author: Sunil K Mishra, Department of Prosthodontics, Rama Dental College, Hospital and Research Centre, Kanpur, Uttar Pradesh, India, Phone: +91 7697738478, e-mail: sunilmsr200@yahoo. co.in

How to cite this article: Mishra SK, Chowdhary R. Current Evidence on Complete Digital Workflow in Implant Prosthodontics. Int J Prosthodont Restor Dent 2021;11(3):109.

Source of support: Nil

Conflict of interest: None

Current evidence available has not distinctly favored the complete digital workflow. The decision to follow either a partial or complete digital workflow in partially edentulous patients should be the sole decision of the operator, based on patients' comfort and treatment cost. Complete digital workflows seem to be the future of implant-supported fixed prosthodontics as it reduces the laboratory steps with fewer technical errors; however, more long-term follow-ups clinical trials should be done to establish the potential benefits of this technique.

\section{References}

1. Jung RE, Schneider D, Ganeles J, et al. Computer technology applications in surgical implant dentistry: a systematic review. Int J Oral Maxillofac Implants 2009;24(Suppl):92-109.

2. Siqueira R, Chen Z, Galli M, et al. Does a fully digital workflow improve the accuracy of computer-assisted implant surgery in partially edentulous patients? a systematic review of clinical trials. Clin Implant Dent Relat Res 2020;22(6):660-671. DOI: 10.1111/cid.12937.

3. Blatz MB, Conejo J. The current state of chairside digital dentistry and materials. Dent Clin North Am 2019;63(2):175-197. DOI: 10.1016/j. cden.2018.11.002.

4. Lin CC, Wu CZ, Huang MS, et al. Fully digital workflow for planning static guided implant surgery: a prospective accuracy study. J Clin Med 2020;9(4):980. DOI: 10.3390/jcm9040980.

5. Gintaute A, Weber K, Zitzmann NU, et al. A double-blind crossover RCT analyzing technical and clinical performance of monolithic $\mathrm{ZrO}_{2}$ implant fixed dental prostheses (iFDP) in three different digital workflows. J Clin Med 2021;10(12):2661. DOI: 10.3390/jcm10122661.

6. Michelinakis G, Apostolakis D, Kamposiora P, et al. The direct digital workflow in fixed implant prosthodontics: a narrative review. BMC Oral Health 2021;21(1):37. DOI: 10.1186/s12903-021-01398-2.

(O) The Author(s). 2021 Open Access This article is distributed under the terms of the Creative Commons Attribution 4.0 International License (https://creativecommons. org/licenses/by-nc/4.0/), which permits unrestricted use, distribution, and non-commercial reproduction in any medium, provided you give appropriate credit to the original author(s) and the source, provide a link to the Creative Commons license, and indicate if changes were made. The Creative Commons Public Domain Dedication waiver (http://creativecommons.org/publicdomain/zero/1.0/) applies to the data made available in this article, unless otherwise stated. 